\title{
Dimorfismo sexual en el acebo, Ilex aquifolium: ¿coste de la reproducción, selección sexual o diferenciación fisiológica?
}

\author{
Sexual dimorphism in holly Ilex aquifolium: cost of reproduction, sexual selection or \\ physiological differentiation?
}

JOSÉ RAMÓN OBESO ${ }^{1} \&$ RUBÉN RETUERTO²

\begin{abstract}
${ }^{1}$ Departamento de Biología de Organismos y Sistemas, Unidad de Ecología, Universidad de Oviedo, E-33071 Oviedo, España; e-mail: jrobeso@correo.uniovi.es ${ }^{2}$ Área de Ecología, Facultad de Biología, Universidad de Santiago de Compostela, E-15071 Santiago, España
\end{abstract}

\begin{abstract}
RESUMEN
Se examina la importancia de tres hipótesis que pueden explicar la existencia de dimorfismo sexual en caracteres secundarios (tamaño) en árbol dioico Ilex aquifolium (Aquifoliaceae): coste de la reproducción, competencia entre machos y diferenciación fisiológica entre sexos. Para ello se presenta una síntesis de trabajos publicados junto con nuevos resultados de tipo observacional y experimental. Durante la floración los machos invirtieron el triple de carbono y el doble de nitrógeno que las hembras. En la fructificación, los árboles hembras invirtieron 10 veces más que los machos en carbono, pero sólo cinco veces más en nitrógeno. No se encontraron diferencias entre sexos en la dinámica foliar y las diferencias en el crecimiento de las ramas fueron dependientes del contexto, aunque en general crecieron más en los machos. En periodos de tiempo largos, 10-30 años, el grosor medio de los anillos de crecimiento de los árboles fue superior en los machos. Las ramas en las que se interrumpió la reproducción eliminando los brotes florales crecieron significativamente más que aquellas que maduraron frutos. Se encontraron diferencias entre machos en la producción de granos de polen por flor. La realización de todos los cruces posibles entre cinco machos y cinco hembras demostró fuertes efectos maternos en la fecundidad, pero no hubo diferencias entre machos en la probabilidad de dejar descendencia. Existen diferencias fisiológicas entre sexos en condiciones de baja luminosidad; así la eficiencia de la fotosíntesis (evaluada como Fv' / Fm') fue mayor en las hembras y por el contrario, los machos fueron más eficientes en el uso del agua (evaluado mediante discriminación isotópica del carbono). Finalmente se discute la importancia de las hipótesis consideradas para explicar estos resultados.
\end{abstract}

Palabras clave: anillos de crecimiento, inversión en reproducción, efectos maternos, competencia entre machos, eficiencia de la fotosíntesis, discriminación isotópica de carbono, tasa relativa de crecimiento.

\begin{abstract}
Three hypotheses were examined in order to explain the existence of sexual size dimorphism in the dioecious tree Ilex aquifolium (Aquifoliaceae): cost of reproduction, sexual selection (pollen competition) and physiological differentiation between sexes. Here we present both published and new experimental and observational results. At flowering, males allocated more in reproduction than females; however at fruiting females allocated about ten times more in terms of carbon and about five times more in terms of nitrogen than males. Both sexes did not differ in leaf dynamics. Branch RGR usually showed greater values in male branches, but the results depended on the ecological context. Tree growth rate, estimated from mean width of annual rings over 10-30 yr, was significantly higher for males. Experimental debbuding in reproductive branches of females accounted for an increase in RGR compared to branches that matured fruits. When competition among males was examined, there were significant differences among male trees in pollen production per flower. The experimental crossing of five males and five females demonstrated strong maternal effects on fecundity, while non significant differences among males were found in their probability of sire progeny. There were some physiological differences between sexes. The efficiency of photosynthesis of leaves (measured as Fv' / Fm') on nonfruiting branches of females was higher than for leaves on branches of male trees under low-light conditions. On the other side, the water-use efficiency, measured by carbon isotope discrimination, was greater in males under xeric conditions. These results are used to discuss the importance of the above mentioned hypothesis.
\end{abstract}

Key words: carbon isotope discrimination, maternal effects, male competition, photosynthetic efficiency, relative growth rate, reproductive allocation, tree-ring growth. 


\section{INTRODUCCIÓN}

La evolución de la dioecia ha sido tradicionalmente explicada por dos hipótesis. La primera considera que el factor selectivo más importante es su función como mecanismo que evita la endogamia y la segunda sostiene que la selección sexual ha sido la fuerza selectiva que ha conducido a la especialización sexual (Baker 1984, Freeman et al. 1997). Ambas hipótesis hacen referencia a la evolución del dimorfismo sexual floral, pero la evolución de los caracteres sexuales secundarios ha sido explorada más raramente (véase no obstante Lloyd \& Webb 1977, Geber 1995, Delph 1999).

Machos y hembras pueden estar sujetos a selección direccional divergente debido a diferencias específicas del sexo durante la reproducción (Willson 1991, Kohorn 1994). Las hembras de la mayoría de las especies dioicas invierten proporcionalmente más biomasa y energía que los machos en la reproducción (Lloyd \& Webb 1977 , Delph 1999) y en consecuencia pueden incurrir en mayores costes, lo cual puede ser evaluado en términos somáticos (menor tamaño o tasa relativa de crecimiento, Tuomi et al. 1983) o en términos demográficos (mayor tasa de mortalidad, menor intensidad y frecuencia de los períodos de reproducción y menos propagación clonal, Karlsson et al. 1990). Mientras que la existencia de los costes somáticos puede manifestarse en la aparición de diferencias de tamaño entre ambos sexos, los costes demográficos asociados pueden manifestarse en razones sexuales sesgadas si hay una mortalidad diferencial asociada al sexo. Estas diferencias intersexuales en el coste de la reproducción pueden ser determinantes evolutivos del dimorfismo sexual en caracteres sexuales secundarios que definan ciclos de vida específicos del sexo (Delph 1999).

El dimorfismo sexual en los caracteres sexuales secundarios también puede ser una consecuencia de la selección intra-sexual (o competencia entre machos o competencia polínica) como consecuencia de la variación en los caracteres que afectan el éxito en la fecundación (Geber 1995). Así, los machos de mayor tamaño pueden ser favorecidos por la selección natural cuando compiten por el acceso a las hembras (Darwin 1871, Willson 1994), ya que producen más polen, incrementando la probabilidad de paternidad sobre una progenie más numerosa (Bawa 1980, Sutherland 1987, Broyles \& Wyatt 1990, Emms et al. 1997, pero véase Lloyd \& Webb 1977, Lloyd 1982).

Por otra parte, la aparición de dimorfismo sexual en caracteres sexuales secundarios puede ligarse a diferencias en las presiones selectivas resultan- tes de la ecología específica de cada sexo (Slatkin 1984). Así, en algunos casos se ha demostrado la existencia de una segregación espacial de los sexos a lo largo de gradientes ambientales, atribuida a la competencia inter-sexual (Bierzychudek \& Eckhart 1988). Varios estudios sugieren que los atributos fisiológicos específicos de cada sexo pueden ser decisivos a la hora de explicar las diferencias en el crecimiento, la distribución de recursos y otras propiedades del ciclo vital (Dawson \& Ehleringer 1993, Retuerto et al. 2000).

El acebo Ilex aquifolium (Aquifoliaceae) es una especie dioica arbórea. El estudio de los patrones de crecimiento y reproducción, en dos poblaciones del Norte de España, una en un bosque de crecimiento secundario dominado por acebos (acebeda) y otra en el sotobosque de un hayedo (Fagus sylvatica), reveló la existencia de algunas diferencias entre sexos: (1) las hembras invirtieron más recursos que los machos en la reproducción, en términos de peso seco (Obeso 1996, 1997); (2) el tamaño, estimado como el diámetro de los troncos, fue mayor en los machos en el sotobosque del hayedo, pero no en la acebeda (Obeso et al. 1998); (3) la razón sexual está sesgada a favor de los machos en el sotobosque. El sesgo a favor de los machos se encuentra en ambas poblaciones al considerar por separado cada vástago o pie, así como al considerar la razón sexual de los individuos reproductores (Obeso et al. 1998).

Con esta perspectiva puede suponerse la existencia de un coste de la reproducción, que podría manifestarse con una reducción del crecimiento y la supervivencia en aquel sexo que invierte más en la reproducción. No obstante la existencia de inconsistencias entre las dos poblaciones aconseja examinar las hipótesis alternativas, proponiendo los argumentos a favor y en contra.

Por tanto, el objeto del presente trabajo es evaluar la importancia de las tres hipótesis que pueden explicar la existencia de dimorfismo sexual en los caracteres sexuales secundarios en el acebo: (1) coste de la reproducción, (2) competencia entre machos (selección intra-sexual), (3) diferencias fisiológicas ligadas al sexo. Para ello se presenta una síntesis que considera tanto resultados publicados como datos inéditos. Inicialmente se presenta información sobre la distribución de recursos en la reproducción y posteriormente se presentan observaciones y experimentos en relación con las hipótesis mencionadas.

\section{INVERSIÓN EN REPRODUCCIÓN}

En trabajos previos (Obeso 1997, Obeso et al. 1998) la inversión relativa en la reproducción en el 
acebo fue valorada en términos de peso seco de las estructuras reproductoras frente al de las vegetativas. En general, el peso seco evalúa bien el contenido en carbono, aunque este elemento no suele ser limitante en el crecimiento de las plantas (Reekie \& Bazzaz 1987). El nitrógeno, sin embargo, tiene un impacto fundamental como factor limitante sobre el crecimiento de las plantas (Ågren 1985). Por ello se evaluó la inversión en términos del contenido en nitrógeno en flores, frutos y porción perenne (ramas), procedentes de la población de la acebeda, mediante análisis de $\mathrm{CNH}$.

Ambos sexos mostraron un contenido en $\mathrm{C}$ y $\mathrm{N}$ muy similar en las ramas, tanto en el crecimiento del año como en el resto de la rama (Tabla 1). Durante la floración los machos invirtieron proporcionalmente casi el doble en peso seco o en carbono que las hembras, pero sólo 1,4 veces más si se considera el nitrógeno. En el caso de la flores masculinas más del $30 \%$ del nitrógeno floral se debe al polen, aunque éste supone aproximadamente el $20 \%$ del peso seco de la flor (Tabla 1).

La inversión en la reproducción debe ser examinada desde una perspectiva dinámica para te- ner una visión de la inversión real que puede traducirse en términos de costes (Ashman 1994). Para ello se analizaron flores masculinas poco antes de la antesis y flores marchitas, considerando sus componentes por separado: pedúnculo, perianto y estambres. Así pudo comprobarse que en las flores marchitas sólo se ha perdido el nitrógeno correspondiente al polen, no modificando su composición el resto de los componentes. Por lo tanto, al contrario de lo que ocurre en Sidalcea oregana ssp. spicata (Ashman 1994), no se produce una reabsorción de del nitrógeno invertido en la estructura floral sino que se pierde al marchitarse la flor. Por tanto, en este caso la medida estática que estima la inversión floral puede considerarse próxima a la inversión real en reproducción.

Cuando se consideró el peso seco y el contenido en nitrógeno de los frutos producidos en promedio por rama, las hembras invirtieron en la reproducción seis veces más que los machos en peso seco y cuatro veces más en nitrógeno.

Considerando el árbol como nivel de integración, debe tenerse en cuenta que los machos no

TABLA 1

Peso seco y contenido en carbono y nitrógeno en las ramas de machos y hembras de Ilex aquifolium, considerando las estructuras perennes (rama) y las de reproducción (flores, frutos). Se indica también la inversión en ambos sexos durante la floración y en hembras durante la fructificación, calculado como proporción de recursos (peso seco, carbono o nitrógeno) contenidos en las estructuras reproductoras respecto al peso seco de las estructuras perennes (rama). Se indica media \pm DE $(n)$

Dry mass (mg), carbon and nitrogen content $(\%)$ for branches from male and female holly trees, considering separately perennial (shoot) and reproductive (flowers, fruits) structures. Reproductive allocation, calculated as the ratio of resources (dry mass, carbon, nitrogen) in sexual structures to resources in perennial structures (shoot). Values are mean \pm SD $(n)$

\begin{tabular}{llll}
\hline & $\begin{array}{l}\text { Peso seco } \\
(\mathrm{mg})\end{array}$ & $\begin{array}{l}\text { Carbono } \\
(\%)\end{array}$ & $\begin{array}{l}\text { Nitrógeno } \\
(\%)\end{array}$ \\
\hline $\begin{array}{l}\text { Ramas masculinas } \\
\text { Crecimiento anual } \\
\text { Resto de la rama }\end{array}$ & $256 \pm 152$ & $48,90(3)$ & $1,29(3)$ \\
Ramas femeninas & $2.600 \pm 1.683$ & $48,30(3)$ & $0,56(3)$ \\
Crecimiento anual & $138 \pm 161$ & $47,80(3)$ & $1,13(3)$ \\
Resto de la rama & $2.381 \pm 1.799$ & $48,10(3)$ & $0,64(3)$ \\
Flor masculina & $3,7 \pm 0,6(6)$ & $48,35 \pm 0,34(6)$ & $1,71 \pm 0,14(6)$ \\
Flor femenina & $6,0 \pm 1,0(6)$ & $49,04 \pm 0,64(6)$ & $2,29 \pm 0,10(6)$ \\
Polen, flor-1 & $0,83(6)$ & $54,2(6)$ & $1,22(6)$ \\
Fruto & $143(6)$ & $53,3(6)$ & $0,64 \pm 0,42(30)$ \\
Inversión en la floración & & $0,24 \pm 0,16(30)$ & $0,45 \pm 0,28(30)$ \\
$\quad \begin{array}{l}\text { Machos } \\
\quad \text { Hembras }\end{array}$ & $0,24 \pm 0,16(30)$ & $0,14 \pm 0,08(30)$ & $2,61 \pm 2,20(138)$ \\
$\quad$ Inversión en la fructificación & $0,13 \pm 0,08(30)$ & $1,51 \pm 1,26(138)$ & \\
$\quad$ & $1,45 \pm 1,22(138)$ & & \\
\hline
\end{tabular}


sólo producen más flores por rama $(82 \pm 50$ flores rama $^{-1}$ frente a $25 \pm 13$ flores rama ${ }^{-1}$ en las hembras), sino que además tienen un mayor porcentaje de ramas con flores $(81 \pm 15 \%$ frente al $59 \pm 26$ $\%$ para las hembras). De este modo los machos invierten durante la floración el doble en nitrógeno y el triple en carbono con relación a las hembras. En la fructificación las hembras habrían invertido cinco veces más en nitrógeno y diez veces más en carbono que los machos en la floración.

\section{COSTE DE LA REPRODUCCIÓN}

Como consecuencia de la inversión en la reproducción los costes deberán manifestarse en una o más de las siguientes características del ciclo vital: Crecimiento modular, crecimiento integrado, supervivencia, propagación clonal y frecuencia e intensidad de la reproducción futura.

\section{Diseños observacionales}

Teniendo en cuenta la organización jerárquica de los organismos modulares, los costes somáticos en términos de crecimiento o supervivencia podrían manifestarse a nivel de hoja, rama o individuo.
Si se considera el módulo rama, en general, el crecimiento relativo de las ramas de los machos es superior al de las hembras, especialmente en el caso de ramas con frutos (Obeso 1997). No obstante, los patrones de crecimiento varían entre localidades (Tabla 2). Si se analiza el crecimiento integrado, estimado por la anchura de los anillos de crecimiento del tronco, los machos crecen en promedio significativamente más que las hembras en ambas localidades cuando el período considerado es de 10 años o más (Obeso 1997, Obeso et al. 1998). La anchura media de los anillos de crecimiento en los últimos 30 años para 10 árboles de cada sexo en cada localidad, en la acebeda fue de $0,948 \pm 0,89 \mathrm{~mm}$ por año para los machos y sólo $0,656 \pm 0,100$ para las hembras. En el sotobosque del hayedo se obtuvieron $0,881 \pm 0,124 \mathrm{~mm}$ por año para los machos y 0,580 $\pm 0,76$ para las hembras. Sin embargo, estas diferencias no son observables si se utiliza el crecimiento anual en años concretos (Obeso et al. 1998). Por el contrario, en árboles prereproductores, las hembras crecieron más en diámetro que los machos (Retuerto et al. 2000).

\section{Diseños experimentales}

Un experimento de eliminación de los brotes florales realizado en la acebeda en 1996 sobre ramas reproductoras de machos y hembras indicó que

TABLA 2

Crecimiento (media \pm EE) de las ramas (tasa relativa de crecimiento por año) de machos de ramas no reproductoras de hembras y de ramas que producen frutos, en diferentes años en dos poblaciones: sotobosque de hayedo y acebeda. ( $n=30$ ramas en 1993, n = 24 ramas en 1994 y $1995, \mathrm{n}=25$ en 1996). Grupos seguidos por letras distintas son significativamente diferentes

$$
(\mathrm{P}<0,05)
$$

Annual relative growth rate (mean $\pm \mathrm{SE}$ ) for branches from males, nonfruiting female branches and fruiting branches at two different sites during different years $(n=30$ branches in 1993, $n=24$ in 1994 and 1995, $n=25$ in 1996). Values followed by different letters are significantly different at $\mathrm{P}<0.05$

\begin{tabular}{lccc}
\hline Año y población & Machos & Hembras vegetativas & Hembras con frutos \\
1993 & & & \\
Sotobosque & $0,144 \pm 0,013$ (a) & $0,069 \pm 0,008$ (b) & $0,050 \pm 0,005$ (b) \\
Acebeda & $0,171 \pm 0,019$ (a) & $0,158 \pm 0,003$ (a) & \\
1994 & & & \\
Sotobosque & $0,082 \pm 0,008$ (a) & $0,032 \pm 0,004$ (b) \\
Acebeda & $0,082 \pm 0,011$ (a) & $0,088 \pm 0,013$ (a) & \\
1995 & & & \\
Sotobosque & $0,125 \pm 0,024$ (a) & $0,041 \pm 0,005$ (b) & $0,048 \pm 0,009$ (b) \\
Acebeda & $0,130 \pm 0,015$ (a) & $0,131 \pm 0,017$ (a) & \\
1996 & & & 0,011 (b) \\
Acebeda & $0,098 \pm 0,058$ (a) & & $0,075 \pm 0,055$ (b) \\
\hline
\end{tabular}


las ramas en las que se eliminaron los brotes tuvieron una tasa de crecimiento relativo mayor que las ramas que continuaron con su asignación reproductora, tanto en machos como en hembras (Fig. 1). Además de un efecto del tratamiento también se detectó un efecto del sexo, ya que las ramas de los machos crecieron más que las de las hembras. Estos resultados apoyan la hipótesis de que los costes de la reproducción pueden expresarse a nivel somático.

En un experimento que combinó la defoliación de ramas con el anillado (eliminación de un anillo de corteza que interrumpe la circulación floemática en la rama) se encontró que la defoliación no tuvo efecto significativo sobre la producción de frutos (Fig. 2). Sin embargo, la defoliación en ramas anilladas sí provocó una disminución del peso de frutos producido por rama (Fig. 2).

\section{Resultados en contra}

Cuando se examina el nivel modular correspondiente a las hojas se distinguieron la edad media de las hojas (determinada por las escalas de crecimiento) y mortalidad de las hojas (determinada

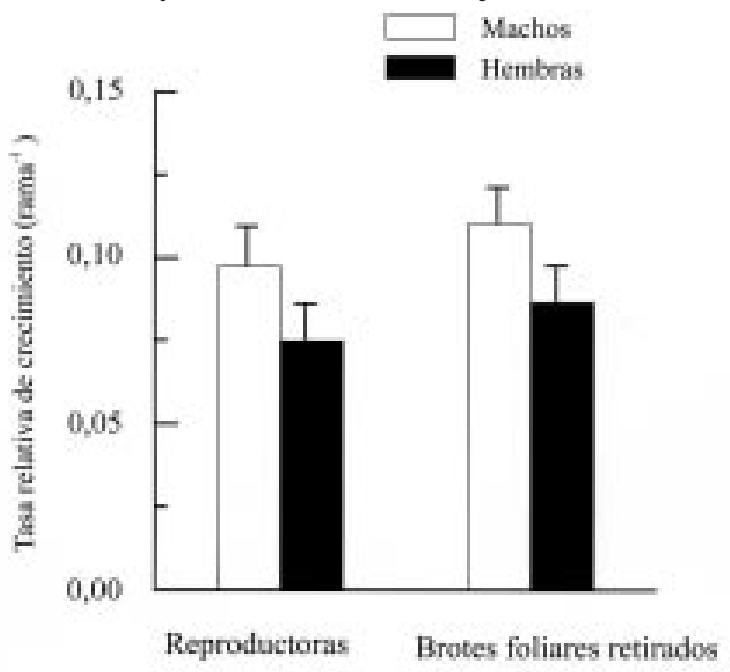

Fig. 1: Tasa relativa de crecimiento (media $\pm \mathrm{EE}$, $\mathrm{n}=24$ ) durante 1996 en la población de la acebeda en ramas de acebos macho y hembra, comparando ramas reproductoras con ramas a las que se les eliminó experimentalmente los brotes florales. Se encontraron efectos significativos del sexo $\left(\mathrm{F}_{1.93}=272,43 ; \mathrm{P}<0,05\right)$ y del tratamiento $\left(\mathrm{F}_{1,93}=385,17 ; \mathrm{P}<0,05\right)$.

Mean branch relative growth rate $( \pm S E, n=24)$ for males and females during 1996, considering fruiting branches and debudded branches. There were significant effects of sex $\left(\mathrm{F}_{1,93}=272.43, \mathrm{P}<0.05\right)$ and experimental treatment $\left(\mathrm{F}_{1,93}\right.$ $=385.17, \mathrm{P}<0.05)$.

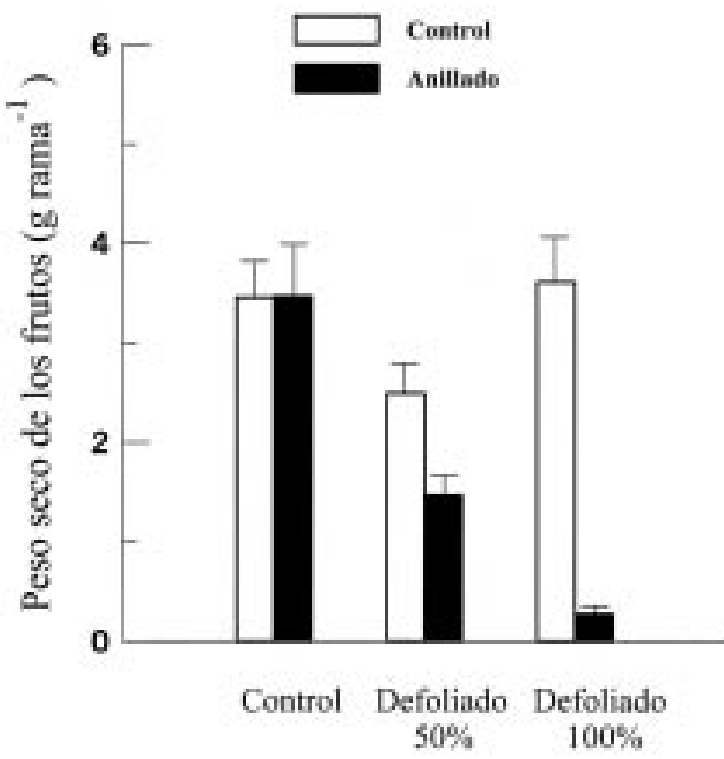

Fig. 2: Efectos de la defoliación (control, defoliado al $50 \%$, defoliado al $100 \%$ ) y del anillado de la rama (control vs. anillado) en la producción de frutos (g peso seco, media \pm EE, $n$ =30) como prueba de la existencia de traslocación de recursos. El efecto del experimento de anillado fue significativo $\left(\mathrm{F}_{1,9}=33,87 ; \mathrm{P}<\right.$ $0,01)$.

Mean $( \pm \mathrm{SE}, \mathrm{n}=30$ ) dry mass of fruits per branch at different defoliation (control, $50 \%$ defoliation, $100 \%$ defoliation) and girdling (control, girdled) treatments. Girdling effects resulted significant $\left(\mathrm{F}_{1,9}=33.87, \mathrm{P}<\right.$ $0.01)$.

por las cicatrices dejadas). No se encontraron diferencias significativas entre sexos para ninguna de las variables (Obeso 1997).

Una manifestación del coste debería ser el compromiso entre crecimiento y reproducción dentro del módulo considerado. Tal sería el caso de la limitación en el crecimiento relativo de una rama impuesto por la producción de flores o frutos. Sin embargo, no se encontró correlación negativa entre la tasa de crecimiento relativo de las ramas y la producción de frutos en las mismas, aunque sí parece existir un límite superior a la inversión global en peso seco (Obeso 1997).

Respecto a la supervivencia de los individuos, no se detectaron diferencias significativas entre sexos y tampoco se encontró segregación espacial de los sexos (Obeso et al. 1998).

\section{COMPETENCIA ENTRE MACHOS}

Si el tamaño de los machos está relacionado con su probabilidad de dejar descendencia, la selec- 
TABLA 3

Resultados del ANOVA jerarquizado que considera la variación en la producción de polen (partículas entre 20 y $25 \mathrm{~mm}$ ) en machos de dos localidades; (**) $\mathrm{P}<0,01 ;$ (***) $\mathrm{P}<0,001$;

(NS) no significativo

Results of the nested ANOVA for pollen production (particles between 20 and $25 \mathrm{~mm}$ ) by males and distribution of variance at locality, tree, and flower level; (**) P $<0.01$; (***) $\mathrm{P}<0.001$; (NS) no significant

\begin{tabular}{lccc}
\hline Fuente de variación & Grados de libertad & Significación & Porcentaje de la varianza \\
\hline Localidad & 1 & NS & 0 \\
Árbol (localidad) & 16 & $* *$ & 39,85 \\
Flor (Árbol (localidad)) & 36 & $* * *$ & 54,02 \\
Error [= réplicas (flor (árbol (localidad))) & 108 & & 6,13 \\
\hline
\end{tabular}

ción intra-sexual o competencia entre machos podría provocar la evolución del dimorfismo sexual en tamaños. Los rasgos potencialmente más importantes como determinantes de la probabilidad de paternidad de un macho podrían ser la longevidad, que indica el número de episodios de reproducción, y el tamaño, que controla el número de flores por episodio de reproducción y probablemente la atracción de polinizadores así como un período de floración más extendido.

Las posibles diferencias en "fitness" entre machos suelen evaluarse mediante indicadores indirectos: (1) producción de flores, (2) producción de polen, (3) tamaño del polen en relación con la probabilidad de paternidad, (4) calidad del polen en relación con la probabilidad de paternidad.

En este caso se examinó, mediante un contador de partículas, el número de granos de polen en tres flores de cada uno de los nueve machos seleccionados aleatoriamente en cada una de las dos localidades. De cada flor se examinó una antera y se realizaron tres repeticiones de cada conteo para determinar el error del método. Se consideraron las partículas dentro del rango de tamaños de los granos de polen de acebo, previamente medidos al microscopio. Se encontraron diferencias notables entre machos en el número de granos de polen que producen por cada antera. La distribución de la variabilidad del sistema examinado (Tabla 3 ) indica que no existen diferencias entre localidades en la producción de polen, casi el $50 \%$ de la varianza total se debe a diferencias entre machos y existe una componente muy importante entre flores dentro de árbol. El error, que examina el efecto de las repeticiones de conteos del número de partículas en una misma antera, es de escasa importancia (Tabla 3).

No se obtuvieron diferencias significativas entre machos cuando se comparó la inversión relativa en flores a nivel de rama. Sólo aparecen diferencias significativas entre sexos, ya que los machos invierten más durante la floración (Tabla 4). Estos resultados probablemente varían a nivel de árbol, debido a diferencias obvias en tamaño y número de ramas con flores.

Las posibles diferencias entre machos en cuanto a su probabilidad de paternidad se examinaron realizando todos los cruces posibles entre cinco hembras y cinco machos, polinizando flores re-

TABLA 4

Resultados del ANOVA que examina las diferencias entre árboles o clones jerarquizados dentro de sexo en la inversión absoluta y relativa, por rama, en peso seco en la floración (peso seco de las flores / peso seco de la rama); (**) $\mathrm{P}<0,01 ;$ (NS) no significativo

Results of nested ANOVAs for dry mass of flowers per branch and relative allocation to flowering comparing sexes and trees within sex; (**) $\mathrm{P}<0.01$; (NS) non significant

\begin{tabular}{lccc}
\hline Fuente de variación & Grados de libertad & Peso seco de las flores & Inversión relativa \\
\hline Sexo & 1 & $* *$ & $* *$ \\
Árbol (sexo) & 9 & NS & NS \\
Error (= entre ramas [árbol $\{$ sexo\}]) & 40 & & \\
\hline
\end{tabular}


TABLA 5

Resultados de los ANOVAs que examinan los efectos de los factores aleatorios materno (5 ind) y paterno ( 5 ind) en la producción de frutos y en las características de los mismos y las semillas; $(* *) \mathrm{P}<0,01 ;(* * *) \mathrm{P}<0,001 ;(\mathrm{NS})$ no significativo

Results of the ANOVAs examining maternal and paternal effects on fruit set, fruit dry mass, pyrenes per fruit, dry masses of pyrenes, seeds and fruit pulp. (**) $\mathrm{P}<0.01$; (***) $\mathrm{P}<0.001$; (NS) non significant

\begin{tabular}{llll}
\hline Fuente de variación & Madre & Padre & Madre x padre \\
\hline Frutos/flores & $* *$ & NS & NS \\
Peso seco del fruto & $* * *$ & NS & NS \\
Pirenos fruto -1 & $* * *$ & NS & NS \\
Peso seco de los pirenos & $* * *$ & NS & NS \\
Peso seco de las semillas & $* *$ & NS & NS \\
Peso seco de la pulpa & $* * *$ & NS \\
\hline
\end{tabular}

ceptivas con polen de cada macho en tres ramas diferentes por cada hembra. Tras el examen de la proporción de frutos iniciados y madurados, no se encontraron diferencias significativas entre padres en la probabilidad de dejar descendencia (proporción de flores polinizadas que llegan a fruto). Sin embargo, sí hubo un importante efecto materno tanto en la probabilidad de que un fruto llegue a madurar como en su tamaño y el de sus componentes (Tabla 5).

\section{DIFERENCIAS FISIOLÓGICAS LIGADAS AL SEXO}

\section{Tasas fotosintéticas}

Se estimó la eficiencia de la fotosíntesis mediante un fluorómetro PAM, a través de la medida de la eficiencia de captura de energía por el fotosistema II, valorada mediante el parámetro de producción Fv' / Fm'. Se midió en hojas de machos y hembras, considerando separadamente ramas que estaban madurando frutos y ramas sin frutos. Aunque en condiciones de saturación de luz no hubo diferencias, en condiciones de baja intensidad lumínica la fotosíntesis resultó más eficiente en las ramas sin frutos de las hembras que en las ramas de los machos (Obeso et al. 1998, Fig. 3).

Eficiencia en el uso del agua evaluada mediante la discriminación isotópica del carbono

En un estoma abierto la concentración de $\mathrm{CO}_{2}$ en el interior $\left(\mathrm{C}_{\mathrm{i}}\right)$ es casi igual que la del aire $\left(\mathrm{C}_{\mathrm{a}}\right)$, mientras que en un estoma cerrado será mucho menor. Puesto que el ${ }^{13} \mathrm{C}$ difunde peor que el ${ }^{12} \mathrm{C}$, en un estoma cerrado con difusión limitada aún

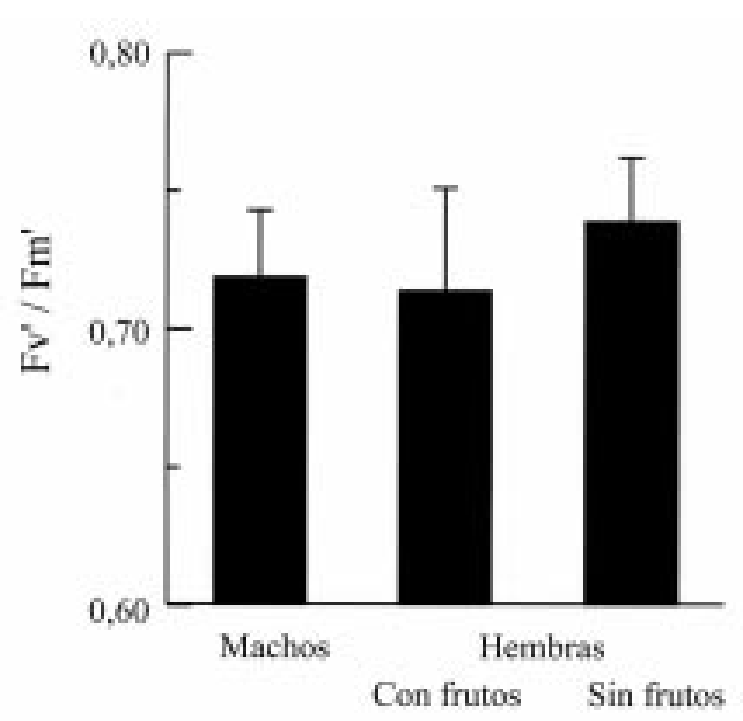

Fig. 3: Eficiencia del fotosistema II en condiciones de baja intensidad lumínica (la radiación fotosintéticamente activa que llegó a las hojas fue de 5-9 $\left.\mathrm{mmol} \mathrm{m}^{-2} \mathrm{~s}^{-1}\right)$, evaluado mediante el parámetro (Fv' / Fm', media $\pm \mathrm{EE}, \mathrm{n}=13$ ) en ramas de machos y en ramas de hembras madurando frutos y vegetativas. Las hojas de las ramas de hembras sin frutos presentaron valores mayores que las de los machos $\left(\mathrm{F}_{1,28}=5,276 ; \mathrm{P}<0,05\right)$.

Efficiency of the photosystem II under low-light intensities ( 5 to $9 \mathrm{mmol} \mathrm{m}^{-2} \mathrm{~s}^{-1}$ ), assessed by the yield parameter ( $\mathrm{Fv}^{\prime} /$ $\mathrm{Fm}^{\prime}$, mean $\pm \mathrm{SE}, \mathrm{n}=13$ ) on leaves from male branches, fruiting branches and non-fruiting branches from females. Fv' / Fm ' values of leaves on nonfruiting branches were significantly higher than for leaves on male branches $\left(\mathrm{F}_{1,28}\right.$ $=5.276, \mathrm{P}<0.05$ ). 
será menor la concentración de ${ }^{13} \mathrm{C}$. Existe por tanto una relación positiva entre proporción de ${ }^{13} \mathrm{C}\left(\mathrm{d}^{13} \mathrm{C}\right)$ y la relación $\mathrm{C}_{\mathrm{i}} / \mathrm{C}_{\mathrm{a}}$. Si hay disponibilidad de agua, los estomas están abiertos y el d ${ }^{13} \mathrm{C}$ (discriminación isotópica) será alto, si hay estrés hídrico el estoma estará cerrado y bajará el valor de $\mathrm{d}^{13} \mathrm{C}$.

Se realizó un diseño experimental de tres factores: sexo (machos, hembras), intensidad lumínica (sol, sombra) y disponibilidad de agua (riego abundante, estrés hídrico) y medimos, entre otras variables la relación isotópica de carbono en los tejidos fotosintéticos producidos durante el experimento. La interacción de tercer orden resultó significativa para el $\mathrm{d}^{13} \mathrm{C}$, indicando que en condiciones de limitación de agua y sin sombra las hembras tienen mayor $\mathrm{d}^{13} \mathrm{C}$, luego mantienen los estomas más abiertos que los machos, siendo por tanto menos eficientes en el uso del agua que aquellos (Fig. 4; Retuerto et al. 2000).

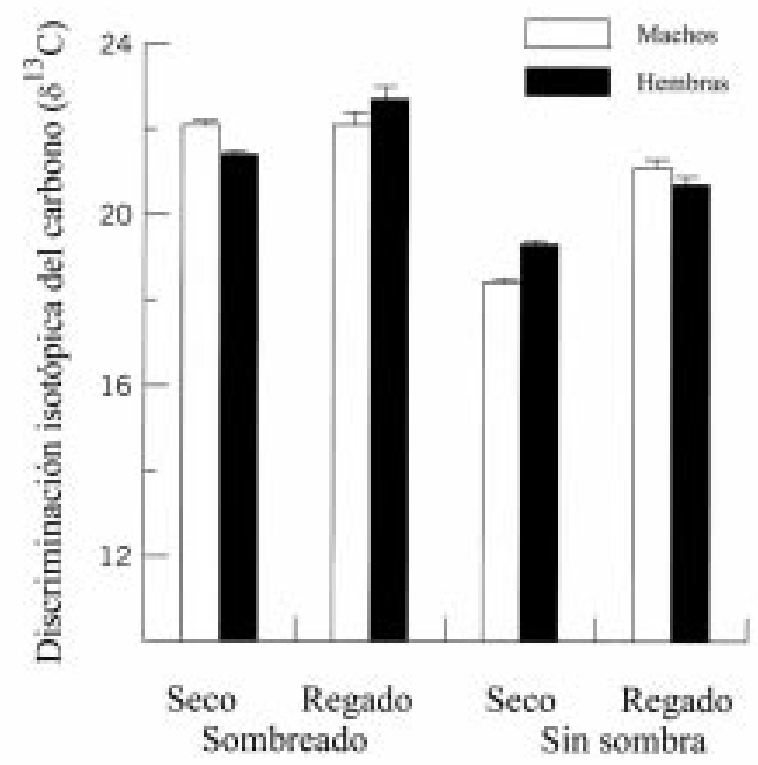

Fig. 4: Discriminación isotópica del carbono $\left(\mathrm{d}^{13} \mathrm{C}\right.$ $\pm \mathrm{EE}, \mathrm{n}=5$ ) en acebos macho y hembra creciendo en diferentes condiciones de sombreo y disponibilidad de agua. La interacción sexo x tratamiento de sombreo $\mathrm{x}$ tratamiento de agua resultó significativa $\left(\mathrm{F}_{1,32)}=11,066 ; \mathrm{P}<0,05\right)$.

Carbon isotope discrimination $\left(\mathrm{d}^{13} \mathrm{C} \pm \mathrm{SE}, \mathrm{n}=5\right)$ for male and female holly trees grown under different light (shaded, unshaded) and water treatments (water limited, well watered). The interaction sex $\mathrm{x}$ light $\mathrm{x}$ water resulted significant $\left(\mathrm{F}_{1,32}=11.066, \mathrm{P}<0.05\right)$.

\section{DISCUSIÓN}

Como es habitual en las especies leñosas dioicas, las hembras de los acebos invierten más en la reproducción que los machos. No obstante la inversión evaluada en peso seco sólo refleja adecuadamente la inversión en carbono, pero no la inversión en nitrógeno, que es proporcionalmente menor a pesar del elevado contenido en nitrógeno que presentan las semillas (Obeso 1998b).

La hipótesis del coste de la reproducción explica algunos de los resultados obtenidos; no obstante, los efectos del coste variaron dependiendo del nivel modular considerado e incluso de la localidad estudiada. Costes variables dentro de especie dependiendo del genotipo, de la luz o de los nutrientes pueden ser habituales (Rekiee \& Bazzaz 1992, Bazzaz 1997).

Aunque no se encontró un compromiso entre inversión en reproducción y crecimiento vegetativo de la rama, la dinámica foliar no difirió entre sexos y las diferencias intersexuales en el crecimiento de las ramas dependieron del contexto, cuando se examinó el crecimiento anual medio integrado en el nivel superior (árbol o clon) durante largos períodos de tiempo se encontraron claras diferencias entre sexos. Éstas pueden ser atribuidas al coste de la reproducción en el marco del ciclo vital, indicando un efecto acumulativo de costes difíciles de encontrar a corto plazo pero ineludibles a largo plazo.

Por otra parte, es posible que no se haya encontrado un coste asociado a la reproducción en todas las situaciones examinadas porque los mecanismos que evitan su aparición son muy adaptativos, ya que existen fuertes presiones selectivas contra los individuos que los manifiestan (Bailey 1992, Obeso 1993). Algunos de estos mecanismos para los que se dispone de información se exponen a continuación: (1) las hembras invierten elevadas cantidades de carbono en la reproducción, pero en proporción el nitrógeno invertido es menor. Si se tiene en cuenta que el carbono puede no ser limitante para el crecimiento de los acebos, vemos cómo los costes en términos de otros nutrientes se reducen al 50 cuando se utiliza el nitrógeno como indicador; (2) se produce un aumento de eficiencia de la fotosíntesis en las ramas que no tienen frutos (Obeso et al. 1998), lo cual podría compensar el mayor gasto que afrontan las ramas que producen frutos; (3) existe la posibilidad de traslocar recursos entre ramas para la maduración de los frutos (Obeso 1998a). Los resultados obtenidos en el experimento de defoliación y anillado pueden interpretarse como que las ramas defoliadas son capaces de obtener fotosintato producido en ramas adyacentes si la circulación de los mismos no está inte- 
rrumpida. El mecanismo estaría fundamentado en la hipótesis fuente-sumidero (Gifford \& Evans 1981, Wardlaw 1990); (4) los frutos verdes muestran actividad fotosintética (Obeso et al. 1998), de forma que presumiblemente pueden contribuir en la producción de al menos una pequeña proporción de los recursos requeridos. Los experimentos de retirada de biomasa fotosintética y anillado de las ramas que impide la movilización de fotosintatos externos indican que esa proporción puede ser cercana al $8 \%$ del peso seco de los frutos producidos por rama; (5) las hembras pueden compensar sus costes reduciendo el período de floración y por tanto los costes de mantenimiento floral, reduciendo la proporción de ramas reproductoras y la frecuencia de la reproducción en las ramas (Obeso 1996). Estas últimas presentarían fluctuaciones bianuales en la frecuencia de reproducción; (6) la capacidad de las hembras pre-reproductoras de crecer más que los machos (Retuerto et al. 2000) no sólo demuestra diferencias innatas entre ambos sexos si no que puede explicar, si mantienen esa capacidad de crecer cuando son reproductoras, su habilidad para reducir los costes de la reproducción.

Las diferencias fisiológicas entre sexos son claras pero dependen del contexto ecológico. Como consecuencia de la mayor eficiencia de los machos en el uso del agua éstos presentan un patrón de uso del agua más conservador lo cual les permite un desarrollo superior en los ambientes más proclives al estrés hídrico. Este es el patrón habitual cuando se han encontrado diferencias entre sexos en este aspecto (Freeman et al. 1976, Dawson \& Bliss 1989, Dawson \& Ehleringer 1993, pero véase Crawford \& Balfour 1983). No obstante, la diferenciación fisiológica entre sexos no condujo en este caso a segregación en el uso del hábitat (Obeso et al. 1998). El dimorfismo sexual en el crecimiento y, en último término, en tamaños sería una de las consecuencias de estas diferencias fisiológicas si la población está en un hábitat en el que pueda producirse estrés hídrico de forma habitual. Las diferencias fisiológicas entre sexos podrían haberse seleccionado independientemente o bien derivarse de respuestas a los costes de la reproducción (Dawson \& Ehleringer 1993) que podría ser el mecanismo subyacente, ya que las hembras deben madurar los frutos durante el verano, que es cuando puede producirse el estrés hídrico. El hecho de que las diferencias entre sexos en el crecimiento del árbol sólo se detecten considerando períodos más o menos largos de tiempo sugiere que durante esos años aumenta la probabilidad de que se produzcan uno o varios años con estrés hídrico prolongado, que influirán de forma notoria en el crecimiento diferencial de ambos sexos.
Las diferencias entre sexos en las tasas fotosintéticas se encontraron en condiciones de limitación de luz, pero debe tenerse en cuenta que la fotosíntesis en el acebo puede saturarse a niveles de irradiancia muy bajos (Peterken \& Lloyd 1967). La diferenciación fisiológica ligada al sexo podría derivarse del hecho de que algunos sistemas génicos implicados en la determinación del sexo también podrían regular los sistemas de producción de hormonas de crecimiento (LebelHardenack \& Grant 1997).

No se han encontrado pruebas de que en los machos estudiados exista variabilidad en la probabilidad de dejar descendientes, por lo que estos resultados no apoyan la hipótesis de la selección sexual. Las diferencias encontradas entre hembras, tanto en la probabilidad de madurar frutos como en las características de estos, son consistentes con los importantes efectos maternos que se detectan en la mayoría de los casos (Roach \& Wulff 1987, Niesenbaum 1999). Tampoco sería una prueba a favor de la competencia intra-sexual el hecho de que exista variabilidad en la producción de polen ya que aunque en algunos casos ésta tiene un origen genético (Schmidtling 1983, Gambier \& Mulcahy 1996), en otros los efectos ambientales pueden ser mayores que los genéticos (Delph et al. 1997) y por tanto no es probable que el proceso de selección sexual pueda utilizar esta variabilidad. Lo mismo ocurre con el tamaño del polen, que no fue examinado en el presente trabajo. Por otra parte, algunos autores indican la existencia de elevadas correlaciones genéticas entre sexos, que harían incrementar el tamaño de las hembras en el caso de que la selección sexual aumentara el tamaño de los machos (Abouheif \& Fairbairn 1997). Luego la existencia de competencia entre machos no tiene porqué traducirse necesariamente en dimorfismo sexual.

Con los resultados presentados no puede rechazarse ninguna de las hipótesis planteadas para explicar la evolución del dimorfismo sexual en tamaño en el acebo. No obstante tampoco existe fundamento para que no puedan funcionar sinérgicamente, tal y como se ha indicado para las diferencias fisiológicas y el coste de la reproducción.

\section{AGRADECIMIENTOS}

Queremos agradecer la invitación de Anna Traveset a participar el la reunión del CYTED de Esporles (Mallorca). E. Cabal, N. Fenández-Calvo, M.J. Bañuelos ayudaron en el trabajo de campo. Los análisis de isótopos de carbono se realizaron en SXAIN (Universidad de A Coruña). Este- 
ban Cabal realizó los análisis de CNH. Los resultados que aquí se exponen se financiaron en el contexto del proyecto de C.I.C.Y.T. PB94-1538, del Ministerio Español de Educación y Ciencia.

\section{LITERATURA CITADA}

ABOUHEIF E \& DJ FAIRBAIRN (1997) A comparative analysis of allometry for sexual size dimorphism: assessing Rensch's rule. American Naturalist 149: 540-562.

ÅGREN G (1985) Limits to plant growth. Journal of Theoretical Biology 113: 89-92.

ASHMAN T-L (1994) A dynamic perspective on the physiological cost of reproduction in plants. American Naturalist 144: 300-316.

BAILEY RA (1992) Why we should stop trying to measure the cost of reproduction correctly. Oikos 65: 349352.

BAKER HG (1984) Some functions of dioecy in seed plants. American Naturalist 124: 149-158.

BAWA KS (1980) Evolution of dioecy in flowering plants. Annual Review of Ecology and Systematics 11: 1539

BAZZAZ FA (1997) Allocation of resources in plants: state of the science and critical questions. En: Bazzaz FA \& J Grace (eds) Plant resource allocation: 1-37. Academic Press, New York, New York.

BIERZYCHUDEK P \& V ECKHART (1988) Spatial segregation of the sexes of dioecious plants. American Naturalist 132: 34-43.

BROYLES SB \& R WYATT (1990) Paternity analysis in a natural population of Asclepias exaltata: multiple paternity, functional gender, and the "pollen-donationhypothesis". Evolution 44: 1454-1468.

CRAWFORD RMM \& J BALFOUR (1983) Female predominant sex ratios and physiological differentiation in artic willows. Journal of Ecology 71: 149-160.

DARWIN C (1871) The descendent of man and selection in relation to sex. John Murray, London, United Kingdom. 528 pp.

DAWSON TE \& LC BLISS (1989) Patterns of water use and the tissue water relations in the dioecious shrub, Salix artica: the physiological basis for habitat partitioning between the sexes. Oecologia 79: 332343.

DAWSON TE \& JR EHLERINGER (1993) Gender-specific physiology, carbon isotope discrimination, and habitat distribution in boxelder, Acer negundo. Ecology 74: 798-815.

DELPH LF (1999) Sexual dimorphism in life history. En: Geber MA, TE Dawson \& LF Delph (eds) Gender and sexual dimorphism in flowering plants: 149-173. Springer-Verlag, Berlin, Germany.

DELPH LF, JOHANNSSON MH \& STAPHENSON AG (1997) How environmental factors affect pollen performance: ecological and evolutionary perspectives. Ecology 78: 1632-1639.
EMMS SK, DA STRATTON \& AA SNOW (1997) The effects of inflorescence size on male fitness: experimental tests in the andromonoecious lily, Zygadenus paniculatus. Evolution 51: 1481-1489.

FREEMAN DC, LG KLIKOFF \& KT HARPER (1976) Differential resource utilization by the sexes of dioecious plants. Science 193: 597-599.

FREEMAN DC, J LOVETT-DOUST, A EL-KEBLAWY, KJ MIGLIA \& ED McARTHUR (1997) Sexual specialization and inbreeding avoidance in the evolution of dioecy. Botanical Review 63: 65-92.

GAMBIER RM \& DL MULCAHY (1996) The association between pollen size and Renner complex in Oenothera villaricae and $O$. picensis ssp. picensis and their hybrids. Theoretical and Applied Genetics 92: 140144.

GEBER MA (1995) Fitness effects of sexual dimorphism in plants. Trends in Ecology and Evolution 10: 222223.

GIFFORD RM \& LT EVANS (1981) Photosynthesis, carbon partitioning, and yield. Annual Review of Plant Physiology 32: 485-509.

KARLSSON PS, BM SVENSSON, BÅ CARLSSON \& KO NORDELL (1990) Resource investment in reproduction and its consequences in three Pinguicula species. Oikos 59: 393-398.

KOHORN LU (1994) Shoot morphology and reproduction in jojoba: advantages of sexual dimorphism. Ecology 75: 2384-2394.

LEBEL-HARDENACK S \& SR GRANT (1997) Genetics of sex determination in flowering plants. Trends in Plant Science 2: 130-136.

LLOYD DG (1982) Selection of combined versus separate sexes in seed plants. American Naturalist 120: 571585.

LLOYD DG \& CJ WEBB (1977) Secondary sex characters in plants. Botanical Review 43: 177-216.

NIESENBAUM RA (1999) The effect of pollen load size and donor diversity on pollen performance, selective abortion, and progeny vigour in Mirabilis jalapa. American Journal of Botany 86: 261-268.

OBESO JR (1993) Does defoliation affect reproductive output in herbaceous perennials and woody plants in different ways? Functional Ecology 7: 150-155.

OBESO JR (1996) Producción de frutos y semillas en Ilex aquifolium (Aquifoliaceae). Anales del Jardín Botánico de Madrid 54: 533-539.

OBESO JR (1997) Costs of reproduction in Ilex aquifolium: effects at tree, branch and leaf levels. Journal of Ecology 85: 159-166.

OBESO JR (1998a) Effects of defoliation and girdling on fruit production in Ilex aquifolium. Functional Ecology 12: 486-491.

OBESO JR (1998b) Patterns of variation in Ilex aquifolium fruit traits related to fruit consumption by birds and seed predation by rodents. EcoScience 5: 463-469.

OBESO JR, M ÁLVAREZ-SANTULLANO \& R RETUERTO (1998) Sex ratios, size distributions and sexual dimorphism in growth in the dioecious tree Ilex aquifolium L. (Aquifoliaceae). American Journal of Botany 85: 1602-1608.

PETERKEN GF \& PS LLOYD (1967) Biological flora of the British isles: Ilex aquifolium L. Journal of Ecology 55: 841-858. 
REEKIE EG \& FA BAZZAZ (1987) Reproductive effort in plants. 2. Does carbon reflect the allocation of other resources? American Naturalist 129: 897-906.

REEKIE EG \& FA BAZZAZ (1992) Cost of reproduction as reduced growth in genotypes of two congeneric species with contrasting life histories. Oecologia 90: 21-26.

RETUERTO R, B FERNÁNDEZ, S RODRÍGUEZ \& JR OBESO (2000) Gender, light, and water effects in carbon isotope discrimination, and growth rates in the dioecious tree Ilex aquifolium. Functional Ecology 14: 529-537.

ROACH DA \& R WULFF (1987) Maternal effects in plants. Annual Review of Ecology and Systematics 18: 209-235.

SCHMIDTLING RC (1983) Genetic variation in fruitfulness in a loblolly pine (Pinus tadea L.) seed orchard. Silvae Genetics 32: 76-80.
SLATKIN M (1984) Ecological causes of sexual dimorphism. Evolution 38: 622-630.

SUTHERLAND S (1987) Why hermaphrodite plants produce many more flowers than fruits: experimental tests with Agave mckelveyana. Evolution 41: 750759.

TUOMI J, T HAKALA \& E HAUKIOJA (1983) Alternative concepts of reproductive effort, cost of reproduction, and selection in life-history evolution. American Zoologist 23: 25-34.

WARDLAW IF (1990) The control of carbon partitioning in plants. New Phytologist 116: 341-381.

WILLSON MF (1991) Sexual selection, sexual dimorphism and plant phylogeny. Evolutionary Ecology 5: 210214.

WILLSON MF (1994) Sexual selection in plants: perspective and overview. American Naturalist 144: S13-S39.

Editor Asociado: R. Medel

Recibido el 30 de abril de 2001; aceptado el 8 de enero de 2002 